\title{
Travel Package Recommendation System: A Literature Review
}

\author{
Himani M. Mishra' ${ }^{1}$ Dr. Ms. V. M. Deshmukh² \\ 1Pursuing Masters of Engineering, Department of Information Technology, Prof. Ram Meghe Institute of Technology and Research, \\ Badnera, Amravati, Maharashtra, India \\ ${ }^{2}$ Head of the Department, Department of Information Technology, Prof. Ram Meghe Institute of Technology and Research, Badnera, \\ Amravati, Maharashtra, India
}

\begin{abstract}
Travelling has become a very well known interest of people now a days, especially when many places own their explicit specialty. It has become a good source of monetary support for many nations. In the era of computers, electronics, information technology, now a day's people who are not aware of peculiarities of places, rely on travel package recommender systems suggested by various sites and applications. Following is the review of work done in this area.
\end{abstract}

Keywords: Travel package recommendation, Recommender systems travelling, Travel schedule recommender

\section{Introduction}

We all humans are different as far as our interests and abilities are concerned. It's not like, that everybody likes painting or everyone is fond of dancing, but yes, many of us have interests in common. Our interests are according to what we like doing more, some people can analyze in a better way than others while others might just learn what they are supposed to learn as it is.In earlier days, while planning to go to a trip people used to ask their friends or family when they were not very much sure about what could be a better plan, but still it was not very satisfying method, so in this era of internet,we came up with a better solution, Recommendation Systems

\section{Literature Review}

\section{Multi-agent based recommender system}

In their report [8] Chen-Shu Wang; Chia-Chuan Yeh; ChunYi Lielaborated that to arrange travel schedule was a classical ill define problem. It lacked structure, was uncertain, and had dynamic complexity. There were two ways to tackle TS arrangement (TSA) problem, which included package tourism provided by ravel agency who arranged entire travelling program. The other was independent tourism in which travelers arranged travelling details themselves after collecting the information. And independent tourism was getting popular, due to its tourism flexibility and customization. To cater for independent tourism customer, online traveler recommender system with particular tourism packages had been already developed by many travel agencies. These recommendations focused on query conditions. However, such recommendation result usually become involve in package tourism advertisements and lack of flexibility and such recommender mechanism could not replicate important word-of-mouse effect about traveling experience. So this concluded that the recommender mechanism should be revised for TSA problem solving.

Hence, this research proposed an intelligence traveling recommender (iTR) system based on commonsense reasoning (CR) algorithm. Intelligence travelling recommender system included two reasoning processes, the first was general reasoning and the second was exception one. ITR consisted of three intelligent agents and three automatic mechanisms enable user constantly refine and revise suggested traveling-schedule by iTR. CR was an appropriate methodology to deal with TSA problem because of CR could replicate human decision process actually.

\section{Collaborative Filtering}

In "CompRec -Trip: A composite recommendation system for travel planning" by Min Xie; Lakshmanan, L.V.S.;

Wood, P.T.[6] had reported their work which was based on the central idea that the usual recommender systems provide users with a list of recommendations and a single item under each recommendation like a book or a CD. However travel planning applications could get benefit from a system which could recommend packages whose items will be in the form of sets or sequences and it could be based on user preferences like budget, location etc. That raised the need for a system which could recommend top-k packages for users to choose from. This paper proposed a novel system, CompRec-Trip, which could automatically generate composite recommendations for travel planning. The system leverages rating information from underlying recommender systems, allows flexible package configuration and incorporates users' cost budgets on both time and money. It had a very good graphical user interface which could let users to customize the returned composite recommendations and took into account external local information.

As Anishya, F.; Kumar M S. proposed in the work [5] "A novel approach for travel package recommendation using Bayesian approach", it was analyzed that, Tourist area season topic model was used in traditional approach which addressed the unique trait along the travel statistics and Users could not get customized travel package because they have to choose from the available packages only. Hence, Tourist Relation Area Season Topic Model is enhanced by creating relationship among the users and the data are semantically classified which provides effective assessment on automatic travel group formation. To find neighboring 


\section{International Journal of Science and Research (IJSR) \\ ISSN (Online): 2319-7064 \\ Index Copernicus Value (2013): 6.14 | Impact Factor (2014): 5.611}

places for the particular area the nearest neighbor method helps. Thus user gets customized package which is more efficient than conventional recommendation system. It's better than even Hybrid recommendation strategy to produce an efficient result to the travelers. The collaborative tagging which mainly works in tagging the neighboring places by predicting the value for the suggested areas and this mechanism helps to capture effective results in the real world recommender system.

\section{Content Aware Recommendation System}

In [7] Interdonato, R.; Romeo, S.; Tagarelli, A.; Karypis, G. explained in their work "Versatile Graph-Based Approach to Package Recommendation" some of the crucial aspects. It's explained in that key aspect in that framework was the exploitation of prior knowledge on the content type models of the packages being generated that express what the users expect from the recommendation task. Packages were learned for each package model, while the recommendation stage was accomplished by performing a Page Rank-style method personalized with respect to the target user's preferences, possibly including a limited budget. This developed method had been tested on a Trip Advisor dataset and compared with some newly proposed method for learning composite recommendations.

Because of the emerging trend in research on recommender systems to design a system which could recommend packages instead of single items which seemed to be a little complicated task because the base data was not easily available, it was limited and highly sparse, along with that user constraints of items, and context had also had to be taken into account. There had been some work on that area, but the application focused on the platform and application domain, so ad hoc solutions were provided which were not applicable on the other domains. This paper proposed an independent package recommendation approach which didn't depend on peculiarities of a particular application domain.

Main factor in this framework was exploitation of knowledge of previous content type models of packages that were being generated which expressed what the users were expecting from the task of recommendation. For each package model, packages were learned, while the recommendation stage was accomplished by performing a Page Rank-style method personalized with respect to the target user's preferences, possibly including a limited budget.

\section{Based on Mobile Crowd Sourced Data}

In [1] Recommending travel packages based on mobile crowd sourced data according to Zhiwen Yu; Yun Feng; Huang $\mathrm{Xu}$; Xing she Zhou preferences for locations is collected from mobile crowd sourced data from location based social network services (LBSNs). A travel package recommendation system was thus proposed to help users make travel plans by leveraging mobile crowd sourced data. Main process proposed was that, it extracted user preferences, discovers points of interest (POIs), and determined location correlations from check-in records. It then generated personalized travel packages by considering user preferences, POI characteristics, and temporal spatial constraints such as travel time and starting location. Jie
Pangs was one of the most popular LBSN's in China on which this system prototype is built and evaluated.

\section{Social Based Recommender System}

In [2]" A Cocktail Approach for Travel Package Recommendation" by Qi Liu; Enhong Chen; Hui Xiong; Yong Ge; Zhongmou Li; Xiang Wu they focused upon study of exploiting online travel information for personalized travel package recommendation. There was always some unique characteristics of travel data which distinguished travel packages from traditional items for recommendation; to identify it was the most critical challenge. So, characteristics of the existing travel packages were analyzed and a tourist-area-season topic (TAST) model was developed. This TAST model represented travel packages and tourists by different topic distributions, where the topic extraction was conditioned on both the tourists and the intrinsic features (i.e., locations, travel seasons) of the landscapes.

Then, based on this topic model representation, a cocktail approach was proposed to generate the lists for personalized travel package recommendation. Furthermore, the TAST model was extended to the tourist-relation-area-season topic (TRAST) model for capturing the latent relationships among the tourists in each travel group. Finally, the TAST model, the TRAST model, and the cocktail recommendation approach were evaluated on the real-world travel package data. The cocktail approach was much more effective than traditional recommendation techniques for travel package recommendation because experimental results show that the TAST model could effectively capture the unique characteristics of the travel data. Also TRAST model could be used as an effective assessment for travel group information, by considering tourist relationships.

\section{Based on Location Based Social Network}

In study by [3] Xinhuan Chen; Yong Zhang; Pengfei Ma; Chao Li; Chunxiao Xing findings given by them were that an Effective tool to provide travel services (e.g., Landscapes selection) for tourists is recommender system . Many recommender systems are based on travel data that are supplied by travel agencies, and provide travel packages from a fixed package set, which bring two challenges for travel package recommender system. One is how to generate more travel packages. The other is how to measure more fine-grained user similarity. In order to address these challenges, a package generation and recommendation framework was developed to help travelers select landscapes. First, a Fuzzy Clustering based Package Generation algorithm (FCPG) was proposed to generate new travel packages to improve the overall recommendation effectiveness and then, Dual Topic Model based Package Recommendation algorithm (DTMPR) was developed. It considered two user-related topics (travel seasons and areas), and provides more fine-grained user similarity measure. Experimental results showed that this framework was better than the state-of-the-art methods.

"Personalized Travel Package with Multi-Point-of-Interest Recommendation Based on Crowd sourced User Footprints "[4] by Yu, Z.; Xu, H.; Yang, Z.; Guo, B went in to the details that People share their locations on location based 


\section{International Journal of Science and Research (IJSR) \\ ISSN (Online): 2319-7064}

Index Copernicus Value (2013): 6.14 | Impact Factor (2014): 5.611

social networks and write their likings and disliking about those places there. By these data i.e. crowd source digital footprints, one could guess user preference to locations. In crowd source digital foot prints each user is connected to many locations. The locations which users share on LBSNs is utilized in generating a travel package recommendation which could help them to make travel plan in an efficient way. The approach utilized data collected from LBSNs to model users and locations, and it determined users' preferred destinations using collaborative filtering approaches. Recommendations are generated by jointly considering user preference and spatiotemporal constraints. To generate travel packages a heuristic search-based travel route planning algorithm was designed. A prototype system was developed which obtained users travel demands from mobile client and thus generated travel package containing multiple points of interest and their visiting sequence. This approach dissipated and improvement in accuracy and diversity according to the experimental results.

\section{Conclusion}

All these recommendation systems provide users with a list that they can check. Most of them offer just One POI, but the travel plans consist of various destinations. It will be better if there could be a system which could consider user preferences and additional attributes. It could have been more helping if there could be a travel plan recommendation system which could contain sequence of locations along with feasible travel routes. There is a lot more to explore and expand in this field, which can ease users who like to explore new places or like travelling a lot.

\section{References}

[1] Recommending travel packages based on mobile crowd sourced data Zhiwen Yu; Yun Feng; Huang Xu; Xing she Zhou Communications Magazine, IEEE Year: 2014, Volume: 52, Issue: 8

[2] A Cocktail Approach for Travel Package Recommendation Qi Liu; Enhong Chen; Hui Xiong; Yong Ge; Zhongmou Li; Xiang Wu Knowledge and Data Engineering, IEEE Transactions on Year: 2014, Volume: 26, Issue: 2

[3] A Package Generation and Recommendation Framework Based on Travelogues Xinhuan Chen; Yong Zhang; Pengfei Ma; Chao Li; Chunxiao Xing Computer Software and Applications Conference (COMPSAC), 2015 IEEE 39th Annual Year: 2015, Volume: 2 IEEE Conference Publications

[4] Personalized Travel Package with Multi-Point-ofInterest Recommendation Based on Crowd sourced User Footprints Yu, Z.; Xu, H.; Yang, Z.; Guo, B. Human-Machine Systems, IEEE Transactions.

[5] A novel approach for travel package recommendation using Bayesian approach Anishya, F.; Kumar M, S. Computing and Communications Technologies (ICCCT), 2015 International Conference.

[6] A composite recommendation system for travel planning Min Xie; Lakshmanan, L.V.S.; Wood, P.T. Data Engineering (ICDE), 2011 IEEE 27th International Conference on Year: 2011
[7] A Versatile Graph-Based Approach to Package Recommendation Interdonato, R.; Romeo, S.; Tagarelli, A.; Karypis, G. Tools with Artificial Intelligence (ICTAI), 2013 IEEE 25th International Conference on Year: 2013 IEEE Conference Publications

[8] Intelligence traveling schedule recommender based on commonsense reasoning algorithm Chen-Shu Wang; "Chia-Chuan Yeh; Chun-Yi Li Computer and Communication Engineering" (ICCCE), 2010 International Conference on IEEE Conference Publications

[9] B. Liu, M. Hu, "Mining and Summarizing Customer Reviews", Proceedings of the ACM SIGKDD International Conference on Knowledge Discovery \& Data Mining (KDD’04), USA.

[10]A. M. Popescu and O. Etzioni: Extracting Product Features and Opinions from Reviewsll, Proceedings of the 2005 Conference on Empirical Methods in Natural Language Processing (EMNLP'05), Canada, 2005. 